\title{
Simulation of Starting and Stopping Vortices of an Airfoil
}

\author{
Roberto Agromayor Jairo Rúa Reidar Kristoffersen \\ Department of Energy and Process Engineering, Norwegian University of Science and Technology, Norway, \\ roberto.agromayorentnu.no
}

\begin{abstract}
When the flow around a streamlined body is accelerated or decelerated,starting and stopping vortices are shed from the trailing edge of the body, respectively. In this work, the transient flow around a NACA4612 airoil profile was analyzed and simulated at $\operatorname{Re}=1000$ and $\alpha=16^{\circ}$ paying especial attention to the starting and stopping vortices shed from the airfoil. A detailed review of the underliying physics of the generation of lift was presented with focus on the importance of viscosity as the essential factor for the generation of lift. The incompressible Navier-Stokes equations with constant density and viscosity in an inertial frame of reference were solved with OpenFOAM using a linear upwind finite volume method (FVM) for the space discretization and the implicit Euler method for the time integration. The results were verified using the Kelvin circulation theorem. Three flow animations were prepared with the simulation results and compared with the historical flow visualizations from Prandtl.
\end{abstract}

Keywords: Kelvin circulation theorem, Stokes theorem, CFD, PIMPLE algorithm, C-mesh, SnappyHexMesh, unsteady, non-inertial, NACA profile, aerofoil.

\section{Introduction}

When the flow around a airfoil starts from rest, either because a free stream velocity is imposed or because the body is set to motion, a starting vortex is formed at its trailing edge. Similarly, when the flow is decelerated or stopped, a stopping vortex of opposite sense is formed and shed from the trailing edge of the airfoil, see Figure 1.

These physical phenomena were recorded in the historical flow visualization by Prandtl. The original flow visualizations are shown in the more recent series of videos of the (National Committee for Fluid Mechanics Films, 1972). The reader is encouraged to watch these flow visualizations because they illustrate the physical phenomena brought to focus in this paper (Vorticity Part 2, 3:00-5:15).

The flow visualizations of Prandtl were analyzed by (Willert and Kompenhans, 2010) using particle image velocimetry (PIV) to compute and visualize the vorticity of the flow. In addition, (Vincent and Blackburn, 2014) performed a direct numerical simulation (DNS) of the transient flow over a symmetrical profile NACA0012 at $\operatorname{Re}=10000$ and $\alpha=4^{\circ}$ using a spectral element method (SEM) that showed the formation of the starting and stopping vortices.
In this work the transient laminar flow over a NACA4612 airfoil profile at $\operatorname{Re}=1000$ and $\alpha=16^{\circ}$ was simulated using a finite volume method (FVM) in OpenFOAM. The three flow visualization by Prandtl: 1) starting flow over an airfoil, 2) impulsively started airfoil in a resting fluid, and 3) impulsively started and stopped airfoil in a resting fluid, were simulated. The lift and drag coefficients were computed as a function of time and the numerical results were verified (Section 3 ) and validated (Section 4) comparing them with the theoretical predictions (Section 2). The purpose of this paper is to study the physics of starting and stopping vortices from a computational point of view and to assess the performance of the OpenFOAM incompressible solvers.

\section{Underlying Physics}

\subsection{The lift and drag forces}

A body immersed in a flow will experience forces from the fluid. In the case of a plane flow, the force parallel to the direction of the free stream direction is known as drag and the force perpendicular the the flow direction is called lift. These forces are the result of the pressure and viscous stresses over the surface of the immersed body.

Lifting bodies, such as airfoils, are designed to provide large forces in the direction normal to the flow and low drag. The performance of a cylindrical airfoil section is characterized by the lift and drag coefficients, defined as:

$$
C_{L}=\frac{D}{\frac{1}{2} \rho V_{\infty}^{2} \cdot c} \quad \text { (1) } \quad C_{D}=\frac{L}{\frac{1}{2} \rho V_{\infty}^{2} \cdot c}
$$

where $L$ and $D$ are the lift and drag forces per unit of width, $\frac{1}{2} \rho V_{\infty}^{2}$ is the dynamic pressure, and $c$ is the chord length of the airfoil profile.

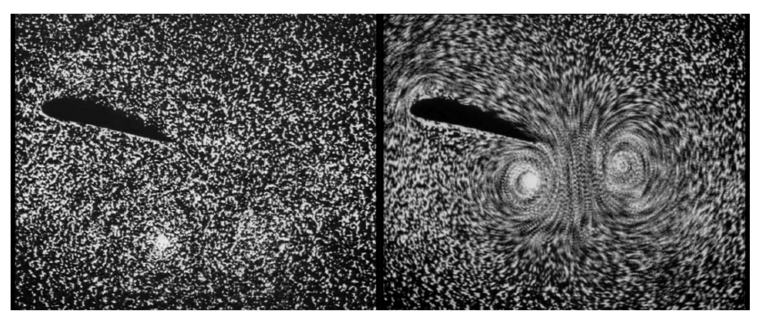

Figure 1. Prandtl's flow visualization. Left: fluid at rest. Right: Interaction of the starting and stopping vortices after the airfoil has been impulsively started and stopped. 


\subsection{Inviscid flow}

One of the main tasks of aerodynamics and hydrodynamics is to determine lift and drag forces. The simplest approach is to analyze the forces caused by the motion of an ideal fluid without viscosity. This kind of flow is known as potential flow and it is based on the assumption that the flow is irrotational, that is, the vorticity of the field is zero everywhere. The vorticity is a kinematic flow variable defined as the curl of the velocity field:

$$
\vec{\omega}=\vec{\nabla} \times \vec{V}
$$

If the zero-vorticity condition is met, then the velocity field can be shown to be the gradient of a potential function that satisfies the Laplace equation. The details of this theory can be found in (White, 2011, Chapter 4 and 8). The solution of this problem is much simpler than the solution of the viscous Navier-Stokes equations and, in many cases, the flow can be solved analytically.

One of the drawbacks of the potential flow theory is that it fails to predict the drag felt by a body moving with constant velocity, relative to the fluid, caused by the viscous stresses (it accounts for drag due to pressure and acceleration though). This is known as the D'Alembert's paradox.

On the other hand, the potential flow theory can be used to predict the lift force on a body as a result of the pressure distribution. One of the most important results of the potential flow theory is the Kutta-Joukowski lift theorem. This theorem states that the lift force per unit of depth experienced by a cylinder with arbitrary shape immersed in a fluid with uniform free-stream velocity $V_{\infty}$ and constant density $\rho$ is given by:

$$
L=-\rho V_{\infty} \Gamma
$$

Where $\Gamma$ is the circulation around the solid body, which is defined as:

$$
\Gamma=\oint_{C} \vec{V} \cdot d \vec{l}
$$

The Kutta-Joukowski theorem is based on the integration of the pressure distribution over the cylinder surface and its proof can be found in (Kundu and Cohen, 2002, Chapter 6).

The integral in Eq. 5 is performed in a counterclockwise sense and the minus sign of Eq. 4 indicates that a negative circulation leads to a positive lift force. Therefore, the main problem to determine the lift force acting on a body of arbitrary geometry is to determine the circulation $\Gamma$.

Despite the usefulness of the potential flow theory to predict lift forces, there is a fundamental inconsistency within it. The lift force in a potential flow is $L=\rho V_{\infty} \Gamma$ but, how does the flow develop the circulation $\Gamma$ ?

Consider the example of an airfoil initially at rest that is moved in a inviscid fluid. At the beginning the velocity is zero everywhere and so is the circulation around the airfoil. When the airfoil starts to move, the fluid flows around the airfoil, but the circulation will still be zero because the vorticity is zero everywhere. This can be explained using the Stokes' theorem to transform the line integral of velocity of Eq. 5 into the surface integral of vorticity. The meaning and the proof of the Stokes' theorem can be found in (Jeffrey, 2001, Chapter 12).

$$
\Gamma=\oint_{C} \vec{V} \cdot d \vec{l}=\iint_{S} \vec{\nabla} \times \vec{v} \cdot d \vec{S}=\iint_{S} \vec{\omega} \cdot d \vec{S}
$$

Since the vorticity is zero everywhere, there is no circulation and hence, the lift force is zero. The inconsistency of the potential flow theory is now apparent, it predicts that the lift force is proportional to the circulation, but at the same time the circulation must be zero because the flow is irrotational.

Kelvin's theorem is an important result related with circulation and vorticity. This theorem states that in an inviscid, constant density flow with conservative body forces, the circulation associated with a closed curve moving with the fluid does not change with time.

$$
\frac{d \Gamma}{d t}=0
$$

In other words, for the case of potential flow in the absence of non conservative forces, the circulation is conserved. The proof and an detailed discussion of the meaning of this theorem can be found in (Kundu and Cohen, 2002, Chapter 5). The main result is that for the case of a real fluid, the presence of viscous stresses in the integration path $C$ leads to changes in the circulation, that in turn lead to lift forces. Therefore, the origin of lift is the presence of viscosity even if the contribution of viscous forces to the lift at steady motion is modest.

\subsection{Viscous flow}

Consider now an airfoil at rest within a stationary viscous fluid that is suddenly accelerated to $V_{\infty}$ at $t=0^{+}$. The arguments presented in this section are briefly considered in (White, 2011, Chapter 7 and 8) and (National Committee for Fluid Mechanics Films, 1972), and discussed in more detail in (Kundu and Cohen, 2002, Chapter 15) and (Batchelor, 2000, Chapter 6).

When the fluid is at rest the velocity and vorticity are zero everywhere and so is the circulation around any closed contour $C$ that contains the airfoil. When the fluid motion starts the viscous boundary layers start to grow close to the surfaces of the airfoil, but at $t=0^{+}$the vorticity is still zero everywhere and the potential solution corresponding to $\Gamma=0$, as shown in Figure 2, still holds. The flow pattern contains two stagnation points, one in the leading edge of the airfoil and the other on the upper side, close $o$ the trailing edge. The trailing stagnation point is not exactly at the trailing edge and the flow turns abruptly at flow the lower side to the upper side at the trailing edge. In fact, the potential flow theory predicts an unphysical infinite velocity at the trailing edge. 


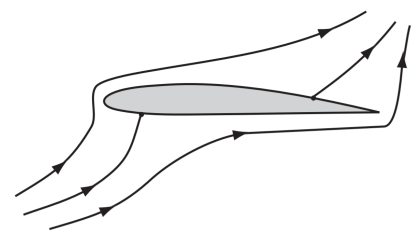

Figure 2. Potential flow solution at $t=0^{+}$for $\Gamma=0$. Figure from (Darmofal, 2001).

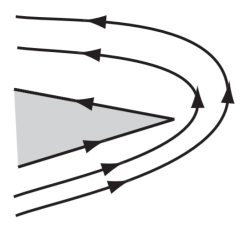

Figure 3. Sharp turn of the potential flow solution at the trailing edge. Figure from (Darmofal, 2001).

The potential flow solution is valid at $t=0^{+}$, even if the fluid has viscosity, because the vorticity is generated at a finite rate close to the airfoil walls and it needs time to be convected and diffused to the main flow. The transport of vorticity in a $2 \mathrm{D}$ incompressible flow is described by:

$$
\frac{\mathrm{D} \vec{\omega}}{\mathrm{D} t}=v \nabla^{2} \vec{\omega}
$$

The left hand side of Eq. 8 is the material derivative of vorticity and the term in the right hand side is diffusion of vorticity due to the viscosity of the fluid. See (White, 2006, Chapter 2) or (Kundu and Cohen, 2002, Chapter 5) for the details of the derivation of this equation.

When $t>0$ the boundary layers start to grow and the vorticity generated close to the walls is transported by the flow. Clockwise vorticity is generated on the upper side of the airfoil while counterclockwise vorticity is created on the lower side of the airfoil. The velocity of the fluid is large at the trailing edge as it flows from the lower to the upper side (see Figure 3) and the flow is decelerated as it approaches the stagnation point. This deceleration causes an adverse pressure gradient that in turn leads to the separation of the boundary layer and backflow. As a result of this backflow, a counterclockwise vortex is formed at the trailing edge of the airfoil. With the passing of time, the stagnation point is displaced closer the the trailing edge and the vortex is convected downstream by the flow. Eventually, the stagnation point occurs at the trailing edge, as pictured in Figure 4 and Figure 5. This situation is known as the Kutta contidion.

The vortex shed from the trailing edge is known as the starting vortex and it is essential in the generation of lift. Once the starting vortex has been shed away from the airfoil we will consider three different integration paths for Eq. 5 as shown in Figure 6. The circulation of the starting vortex is given by the integration around $<\mathrm{efgh}>$. This integral is clearly positive as the vorticity of the starting vortex is positive (counterclockwise) at all points. On the

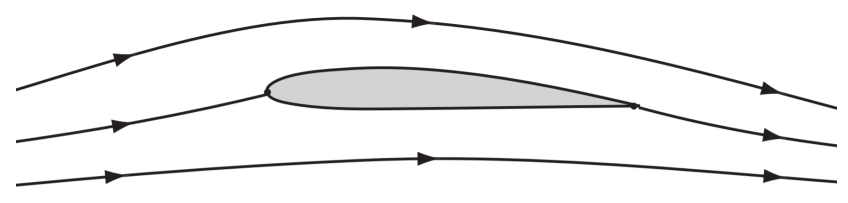

Figure 4. Viscous solution with the the second stagnation point at the trailing edge. Figure from (Darmofal, 2001).

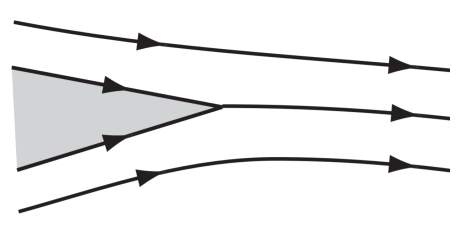

Figure 5. Detail of the Kutta condition with the stagnation point at the trailing edge. Figure from (Darmofal, 2001).

other hand, the circulation around the airfoil (responsible for the lift) is given by the integration around $<$ abcd $>$. The value of both circulations has to be equal in magnitude and opposite in sign, as it will be explained next. Therefore, there is a negative circulation around the airfoil that leads to a positive upwards lift force, see Eq. 4.

The reason why both integrals have to sum zero is explained with Kelvin's circulation theorem. If the circulation integral of Figure 6 is evaluated around an inviscid integration path far away from the airfoil, then the value of the circulation must have remained constant at all times and, since $\Gamma=0$ at $t=0$, the circulation around $<$ aefgcd $>$ must be zero at all times. Finally, since:

$$
\oint_{\text {aefgcd }} \vec{V} \cdot d \vec{l}=\oint_{\text {abcd }} \vec{V} \cdot d \vec{l}+\oint_{\text {efgh }} \vec{V} \cdot d \vec{l}
$$

we can conclude that the the sum of the circulation around the airfoil and the starting vortex must be zero.

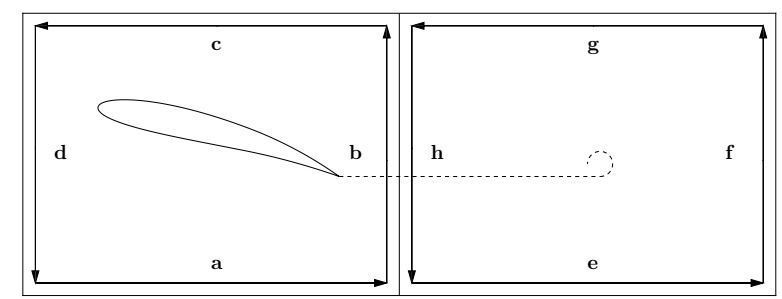

Figure 6. Scheme of the integration paths used to show that the lift is caused by the starting vortex.

\subsection{Starting and stopping vortices}

As it has been discussed, a starting vortex is formed when a flow is started over an airfoil. Likewise, if the free stream velocity is increased, the flow around the airfoil will be accelerated leading to a new starting vortex. In contrast, if the free stream velocity is reduced or stopped, a clockwise stopping vortex will be shed from the airfoil. 
The situation when an airfoil starts its motion within a fluid at rest is very similar. In this case a starting vortex is also formed but instead of being convected downstream with the flow, it remains in the same location where it was generated while the airfoil moves away from the vortex. On the other hand, if the vortex is rapidly decelerated a clockwise stopping vortex will also be formed.

In this work, a numerical investigation of the starting and stopping vortices shed by a NACA4612 at an angle of attack $\alpha=16^{\circ}$ was performed for the cases of a started flow, a started airfoil, and a started and stopped airfoil.

\section{Computational Procedure}

\subsection{Mesh generation and verification}

The transient two-dimensional flow over a NACA4612 airfoil with an angle of attack of $16^{\circ}$ was simulated using a finite volume method (FVM) code. A laminar Reynolds number of 1000 was selected for the computations. The geometry of the NACA4612 profile, shown in Figure 7, was generated according to the procedure of the original NACA report presented in (Jacobs et al., 1933).

Two types of meshes were considered in this work. The former was a C-mesh type, which is a multiblock structured mesh that consists of a semicircle grid that follows the geometry of the airfoil on the leading edge, and a rectangular grid that captures the physics on the trailing edge and the wake region. This is one of the main advantages of the C-mesh, as the grid is finer in the regions where the flow requires higher resolution. The later type of mesh was an automatically-generated, unstructured, hexahedral mesh, which concentrates the vast majority of the cells near the surface of the airfoil.

The C-mesh was created in OpenFOAM importing the geometry of the airfoil from MATLAB. The division of the mesh in 6 blocks is shown in Figure 8, allowing it to have different cell density depending on the region. In addition, a grading on the cell size was applied in order to obtain as many cells as possible near the airfoil while having fewer at the borders of the computational domain.

The cell distribution is shown in Figure 9. The high density of cells close to the airfoil provides more accurate computations in the region where the gradients are more pronounced, which is fundamental to capture the vortex generation. The accuracy of the computations is not deteriorated by mesh skewness since the orthogonality of the first cell layers close to the surface is high. Furthermore, a C-mesh with grading on the cell size allows saving computational resources on regions were the physics are not

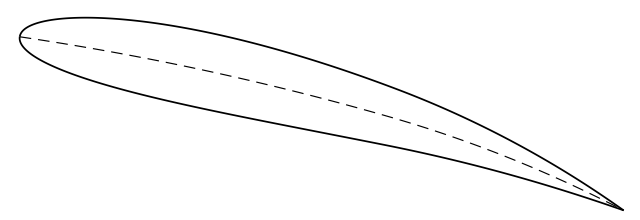

Figure 7. Geometry of the NACA4612 profile at $\alpha=16^{\circ}$.

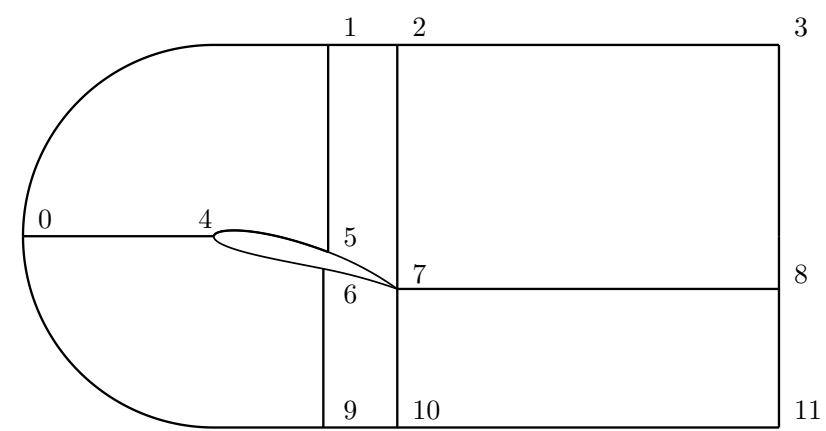

Figure 8. Scheme of the blocks used to generate the structured C-mesh.

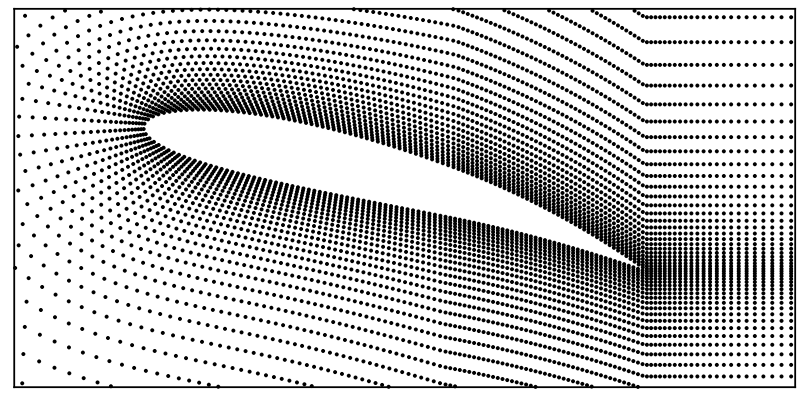

Figure 9. Details of the structured C-mesh close the the airfoil.

relevant, as the inlet, top and bottom of the control volume. Nevertheless, as the main drawback of the C-mesh configuration, some of these regions still have an excessive amount of cells that lengthen the simulation time.

C-meshes with three different dimensions (base case, double size and half size) and number of cells (as seen in Table 1) were tested in order to select the most appropriate one for the simulation and to verify the meshing procedure programmed in MATLAB. For this purpose, steady-simulations at $\operatorname{Re}=100$ were performed with a linear upwind FVM in OpenFOAM using the SIMPLE algorithm for the velocity-pressure coupling. The results for the torque (with respect to the leading edge), drag, and lift coefficients, as well as the computational time and number of iterations are shown in Table 1. The steadystate simulations were performed at $\mathrm{Re}=100$ instead of $\operatorname{Re}=1000$ because at high Reynolds numbers oscillatory vortex shedding occurs and it is not possible to reach a steady-state solution.

The results of the simulation tend to be grid independent, as they gradually converge to the same solution when the grid is refined. In addition, it can be observed that the size of the domain does not affect the results of the simulation and therefore, the solution is independent of the far field boundary conditions (see section 3.2). Thus, due to the similarity among the results, the small mesh with 20000 cells was selected to perform the transient simulations. The reason why the mesh with smallest dimensions was selected is because the shorter computational time it requires for a given level of accuracy. The transient simulations were performed with 20000 cells, even if the re- 
Table 1. C-mesh screening and validation.

\begin{tabular}{ccccccc}
\hline Domain & Cells & $C_{T}$ & $C_{D}$ & $C_{L}$ & Iterations & Time (s) \\
\hline \multirow{6}{*}{ Large } & 5000 & 0.2698 & 0.5167 & 0.7530 & 458 & 10 \\
& 11400 & 0.2690 & 0.5155 & 0.7626 & 796 & 34 \\
& 20000 & 0.2692 & 0.5152 & 0.7675 & 1179 & 103 \\
& 45000 & 0.2707 & 0.5153 & 0.7749 & 2114 & 382 \\
& 80000 & 0.2723 & 0.5157 & 0.7801 & 3238 & 1115 \\
\hline \multirow{6}{*}{ Medium } & 5000 & 0.2739 & 0.5221 & 0.7787 & 464 & 10 \\
& 11400 & 0.2737 & 0.5210 & 0.7825 & 835 & 38 \\
& 20000 & 0.2740 & 0.5207 & 0.7844 & 1264 & 100 \\
& 45000 & 0.2753 & 0.5209 & 0.7892 & 2232 & 389 \\
& 80000 & 0.2767 & 0.5213 & 0.7930 & 3466 & 1115 \\
\hline \multirow{6}{*}{ Small } & 5000 & 0.2847 & 0.5351 & 0.8136 & 591 & 11 \\
& 11400 & 0.2845 & 0.5341 & 0.8143 & 1095 & 40 \\
& 20000 & 0.2846 & 0.5339 & 0.8147 & 1611 & 110 \\
& 45000 & 0.2846 & 0.5340 & 0.8150 & 2788 & 455 \\
& 80000 & 0.2851 & 0.5341 & 0.8163 & 4318 & 1256 \\
\hline \multirow{6}{*}{}
\end{tabular}

sults with a lower number of cells seem accurate. because the authors wanted to ensure that the physics at the trailing edge were captured.

The unstructured mesh is shown in Figure 11. It was created using the snappyHexMesh tool in openFOAM together with the airfoil profile geometry generated in MATLAB. This mesh was validated following the same procedure described before but only for the small domain. The obtained results are summarized in Table 2. The torque, drag and lift coefficients are similar to the ones obtained for the structured mesh, whereas the time and number of iterations needed for convergence are smaller. Thus, it could seem that the unstructured grid is more suitable for the transient simulations since it requires less time to attain a similar solution. However, when the results were post-processed and the velocity and pressure fields were observed in detail, the solution was found to be unphysical as it followed the structure of the grid, even when orthogonal correctors were employed.

The structured C-mesh used in this work was able to capture the physics of the flow around the airfoil better than the unstructured grid. As a result, the $\mathrm{C}$-mesh was chosen for the transient simulations.

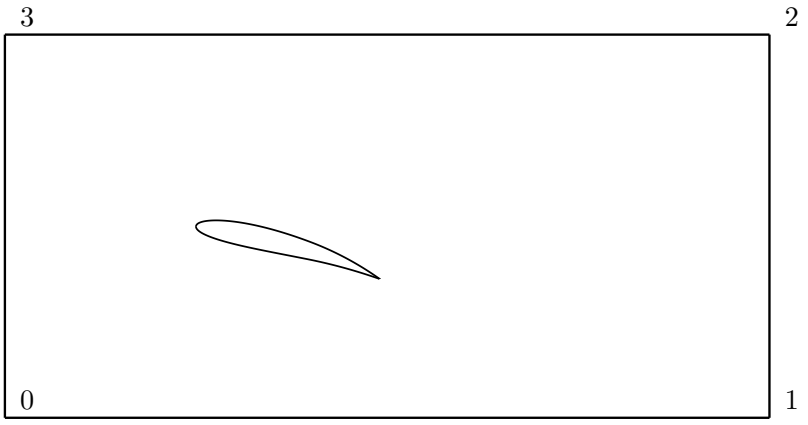

Figure 10. Scheme of the block used to generate the unstructured mesh.
Table 2. Unstructured mesh validation

\begin{tabular}{ccccccc}
\hline Mesh & Cells & $C_{T}$ & $C_{D}$ & $C_{L}$ & Iterations & Time (s) \\
\hline \multirow{4}{*}{ Small } & 3480 & 0.2462 & 0.5115 & 0.7519 & 97 & 1 \\
& 13037 & 0.2574 & 0.5081 & 0.7507 & 193 & 8 \\
& 21988 & 0.2722 & 0.5127 & 0.7867 & 196 & 14 \\
& 32939 & 0.2672 & 0.5087 & 0.7745 & 275 & 97 \\
& 125490 & 0.2797 & 0.5126 & 0.8007 & 303 & 439 \\
\hline
\end{tabular}

Table 3. Boundary conditions in OpenFOAM.

\begin{tabular}{ccc}
\hline Boundary & $\mathrm{U}$ & $\mathrm{P}$ \\
\hline Inlet & tableFile & zeroGradient \\
Outlet & zeroGradient & fixedValue $(0)$ \\
Top & slip & slip \\
Bottom & slip & slip \\
Airfoil & fixedValue $\left(\begin{array}{lll}0 & 0 & 0\end{array}\right)$ & zeroGradient \\
\hline
\end{tabular}

\subsection{Initial and Boundary Conditions}

In order to simulate the motion of the fluid from rest, uniform zero velocity and pressure fields were used as initial conditions. The boundary conditions used in OpenFOAM for the simulations are described in the next subsections and an overview is given in Table 3.

\subsubsection{Inlet boundary conditions}

The velocity field at the inlet boundary is prescribed as a function of time and read from a table. The pressure at the inlet is defined with a zeroGradient boundary condition to simulate the undisturbed far field flow.

The function for the inlet boundary velocity was the same for the simulation of the starting flow over the airfoil and for the simulation of the airfoil that moves within a fluid at rest. The only difference between both cases is a change in the frame of reference. The function chosen for the simulations is given by:

$$
u=V_{\infty} \cdot\left(1+e^{-\frac{t-t_{1}}{T}}\right)
$$

This is a logistic function with an $S$ shape and it was chosen to simulate a very steep jump in velocity with a smooth function in order to avoid numerical problems. The shape of this curve is given in Figure 13. The simula-

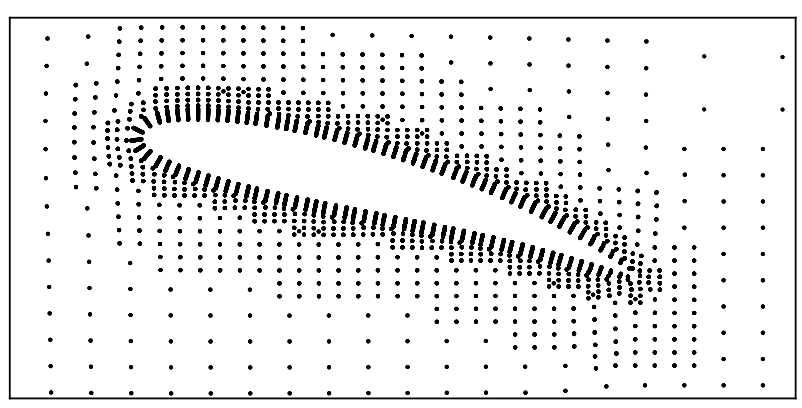

Figure 11. Details of the unstructured mesh close the the airfoil. 
tion time step was set small enough to capture the velocity variation along the jump.

For the case of the airfoil that is suddenly started and then stopped the inlet boundary condition is given by the following function:

$$
u= \begin{cases}V_{\infty} \cdot\left(1+e^{-\frac{t-t_{1}}{T}}\right) & \text { for } t \leq \frac{t_{1}+t_{2}}{2} \\ V_{\infty} \cdot\left(1+e^{\frac{t-t_{2}}{T}}\right) & \text { for } t \geq \frac{t_{1}+t_{2}}{2}\end{cases}
$$

This piecewise smooth function is given by two logistic equations and its shape is shown in Figure 16. The values of the parameters used in all the simulations are $V_{\infty}=1$ $\mathrm{m} / \mathrm{s} t_{1}=0.5 \mathrm{~s}, t_{2}=1.5 \mathrm{~s}$ and $T=0.01 \mathrm{~s}$.

\subsubsection{Airfoil boundary conditions}

A noSlip velocity boundary condition is used at the surface of the airfoil. The velocity of the airfoil is always set to zero during the simulations and its motion is accounted with the inlet boundary conditions. A zeroGradient boundary condition is used for the pressure, this is usually a good approximation for viscous flows.

\subsubsection{Top and bottom boundary conditions}

A slip boundary condition is used for the velocity and pressure fields at the top and the bottom boundaries. This implies zero velocity normal to the boundary and no condition for the tangential direction. The pressure condition was set as a zeroGradient boundary condition. This simulates that the far field behaves as an inviscid fluid.

\subsubsection{Outlet boundary condition}

The velocity at the outlet is defined with a zeroGradient boundary condition to simulate that the flow is fully developed downstream the airfoil. A value of zero is chosen for the pressure field at the outlet to set a reference level for pressure.

\subsection{OpenFOAM solution}

Once the mesh was generated and the boundary conditions were imposed, the incompressible (constant density and viscosity) Navier-Stokes equations were discretized using the linear upwind FVM in space and the implicit Euler method in time and then solved using the PIMPLE algorithm for the velocity-pressure coupling. Once the velocity and pressure fields were computed the results were post-processed to compute the vorticity field as well as the lift and drag coefficients. After that, the results where exported to MATLAB to prepare the flow visualizations.

The Navier-Stokes equations solved in the OpenFOAM PIMPLE algorithm are formulated without body forces in an inertial frame of reference. These equations can be expressed in differential form as:

$$
\begin{gathered}
\nabla \cdot \vec{V}=0 \\
\frac{\mathrm{D} \vec{V}}{\mathrm{D} t}=-\frac{\vec{\nabla} p}{\rho}+v \nabla^{2} \vec{V}
\end{gathered}
$$

For the cases when the airfoil was moving within a fluid at rest, the problem was solved imposing the time-varying boundary conditions on a stationary airfoil and then the results were post-processed to include the motion of the airfoil. The displacement of the airfoil was computed numerically as the integral of the airfoil velocity:

$$
x=\int_{0}^{t} u \cdot d t
$$

The displacement of the airfoil as a function of time is plot in Figure 12 for the case of the suddenly started airfoil and in Figure 15 for the case of the suddenly started and stopped airfoil. However, when the airfoil accelerates from rest, the frame of reference for the relative velocity field is non-inetial and Eq. 14 has to be modified to include the non-inertial effects (fictitious forces):

$$
\frac{D \vec{V}}{D t}=-\frac{\vec{\nabla} p}{\rho}+v \nabla^{2} \vec{V}-\vec{a}
$$

Where $\vec{a}$ is the acceleration of the non-inertial frame of reference (in this case, the acceleration of the airfoil). A throughout derivation and discussion of the inclusion of non-inertial terms into the integral form of momentum equations is presented in (Fox et al., 2011, Chapter 4). As it can be seen from Eq. 16, the acceleration term behaves as a body force (similar to gravity) acting in the direction opposite to the acceleration of the airfoil.

This term was not included into the analysis as the solution algorithm was not prepared to include body forces. In order to asses the importance of this limitation the acceleration was computed numerically as the derivative of velocity and plotted in Figure 14 for the case of the suddenly started airfoil and in Figure 17 for the case of the suddenly started and stopped airfoil.

$$
a=\frac{d u}{d t}
$$

For this velocity distribution the acceleration term peaked at $a \approx 25 \mathrm{~m} / \mathrm{s}^{2} \approx 2.5 \mathrm{~g}$. The gravity term is usually negligible in most aerodynamic applications and, in this problem, the forces due to the acceleration term are of the same order of magnitude as gravity. In addition, the acceleration term is only acting for very short periods of time when the airfoil is impulsively started or stopped. For these reasons, it seems reasonable to neglect the effect of the acceleration in this problem.

\subsection{Dimensionless considerations}

The numerical simulations were performed at a $R e=$ 1000 , this Reynolds number was defined as:

$$
\operatorname{Re}=\frac{V_{\infty} \cdot c}{v}
$$

where: 


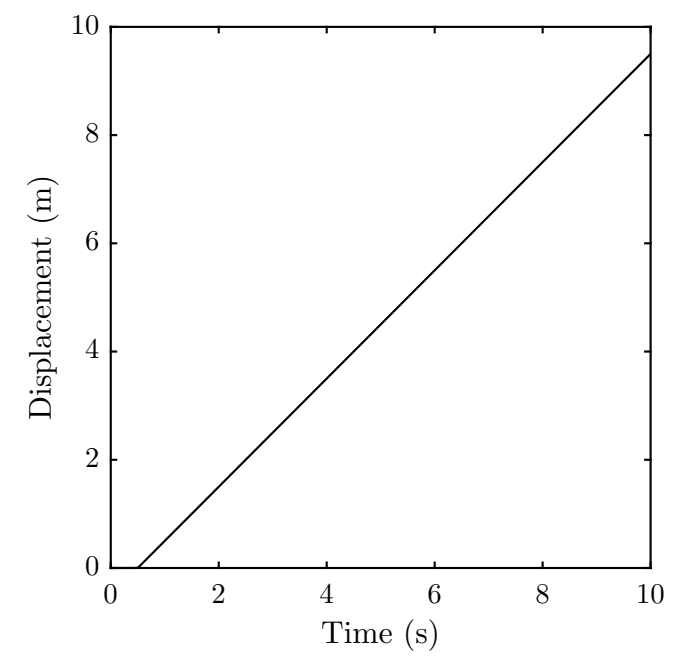

Figure 12. Airfoil displacement for the case when it is impulsively started.

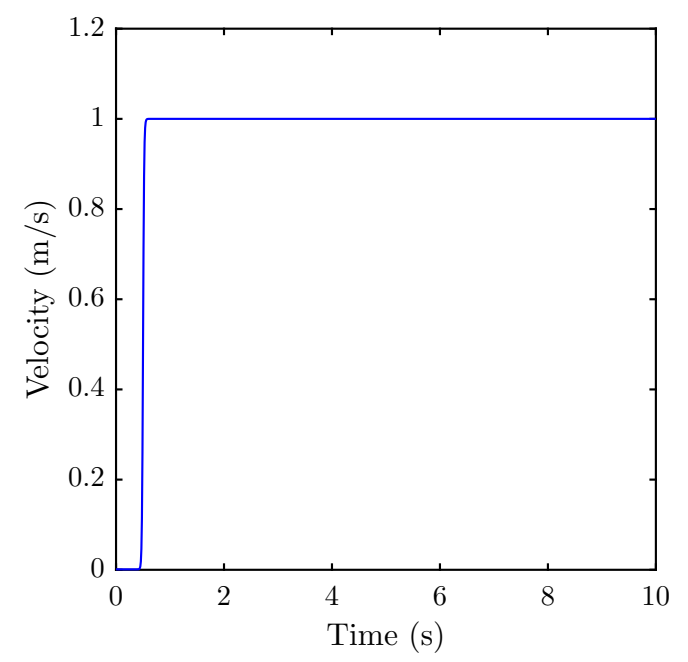

Figure 13. Airfoil velocity for the case when it is impulsively started or free stream velocity for the stationary airfoil.

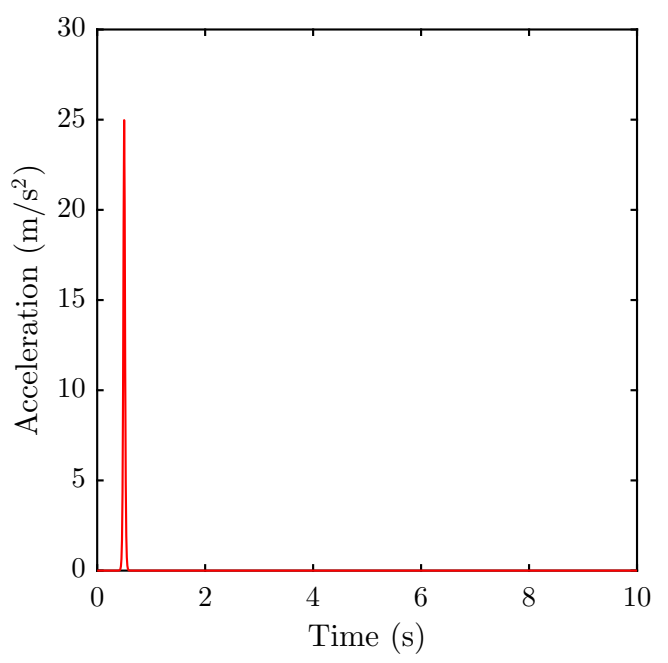

Figure 14. Airfoil acceleration for the case when it is impulsively started.

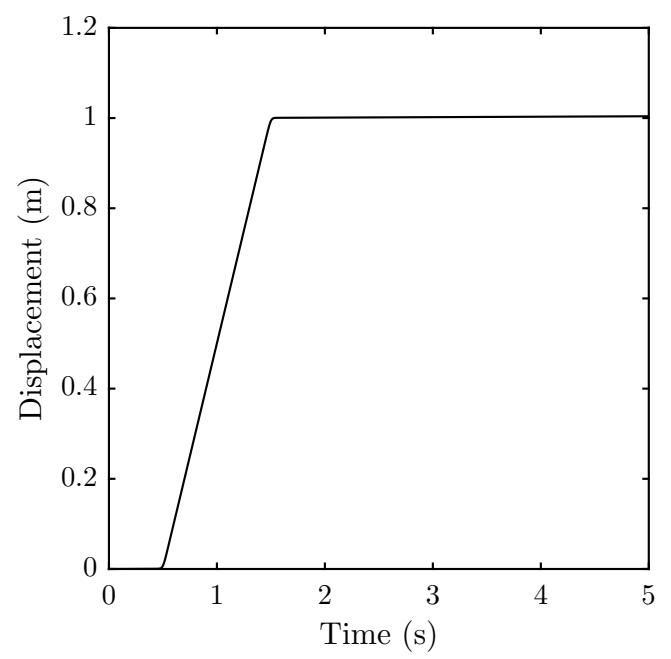

Figure 15. Airfoil displacement for the case when it is impulsively started and stopped.

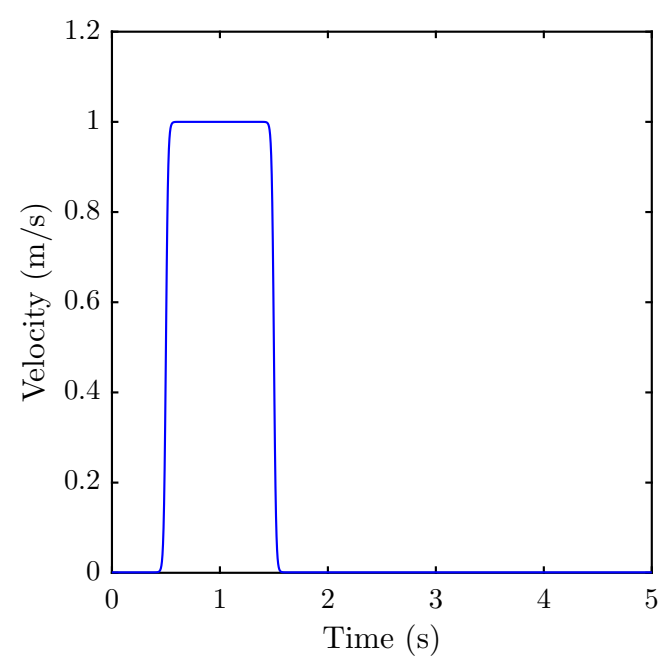

Figure 16. Airfoil velocity for the case when it is impulsively started and stopped.

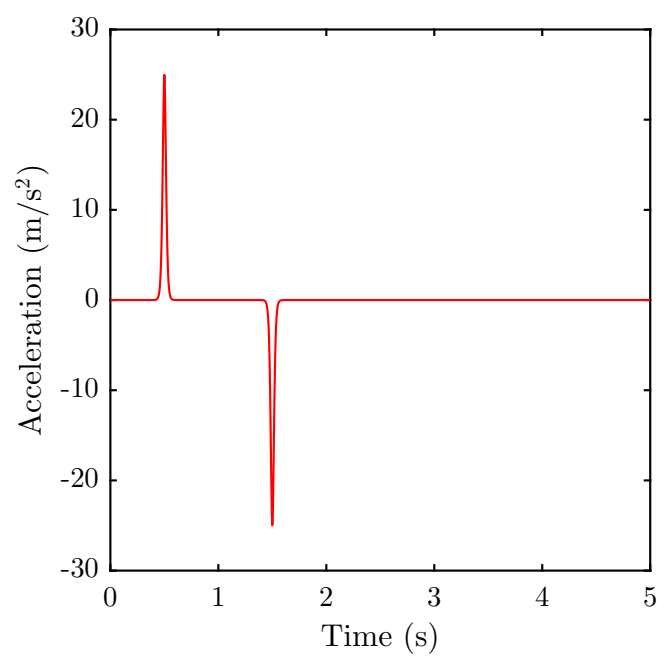

Figure 17. Airfoil acceleration for the case when it is impulsively started and stopped. 


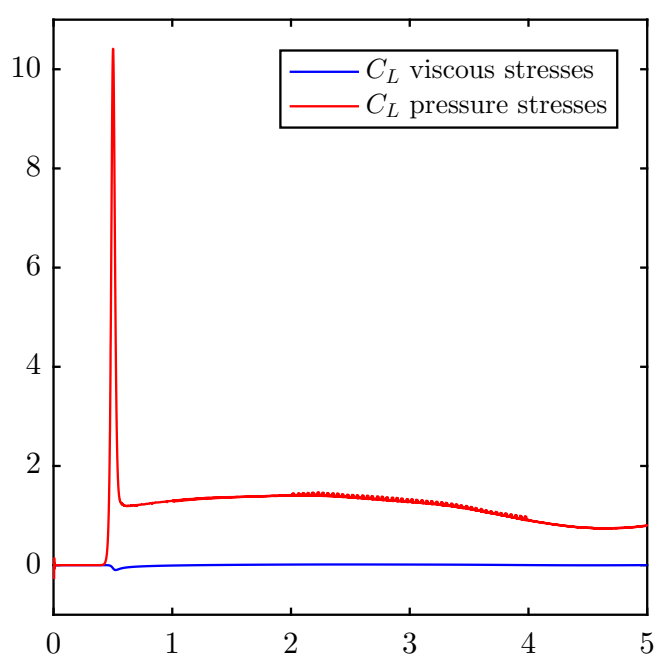

Figure 18. Components of the lift coefficient for the impulsively started airfoil or the stationary airfoil.

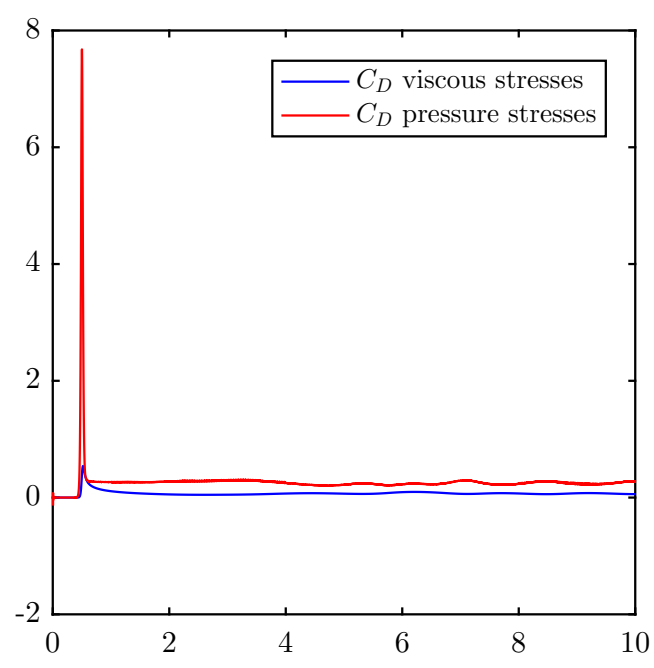

Figure 19. Components of the drag coefficient for the impulsively started airfoil or the stationary airfoil.

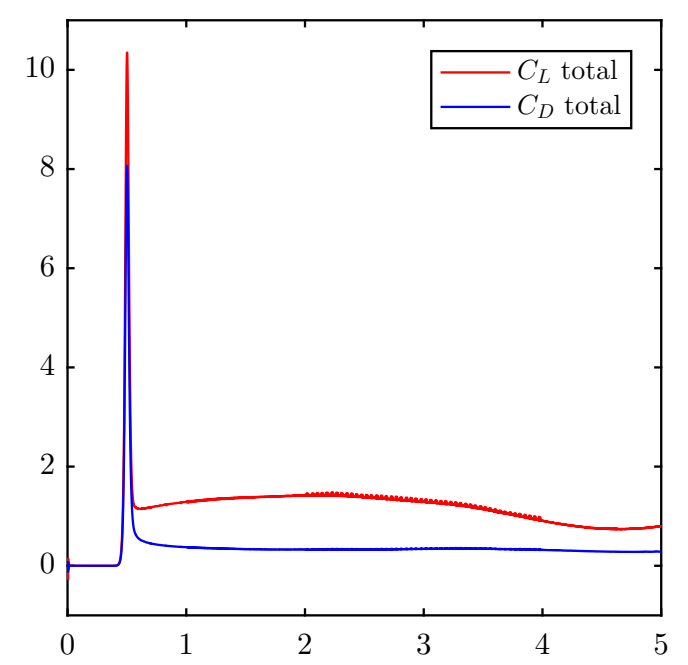

Figure 20. Drag and lift coefficients for the impulsively started airfoil or the stationary airfoil.

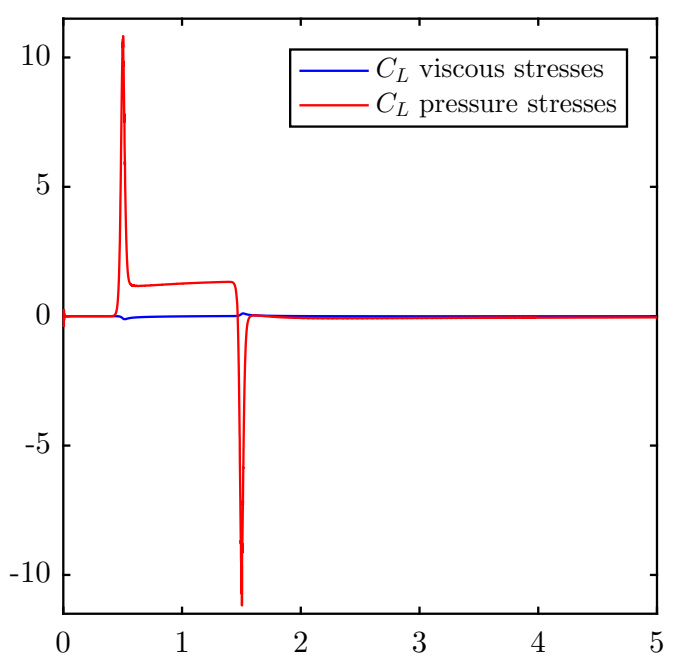

Figure 21. Components of the lift coefficient for the impulsively started and stopped airfoil.

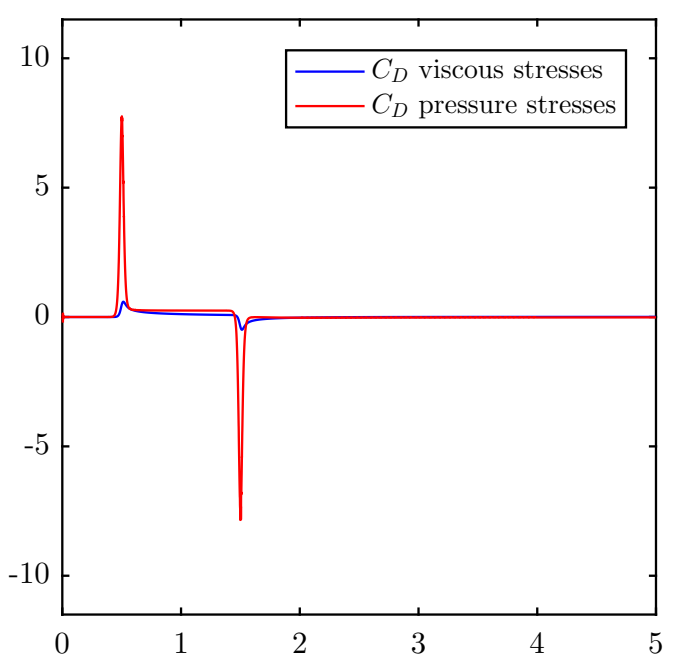

Figure 22. Components of the drag coefficient for the impulsively started and stopped airfoil.

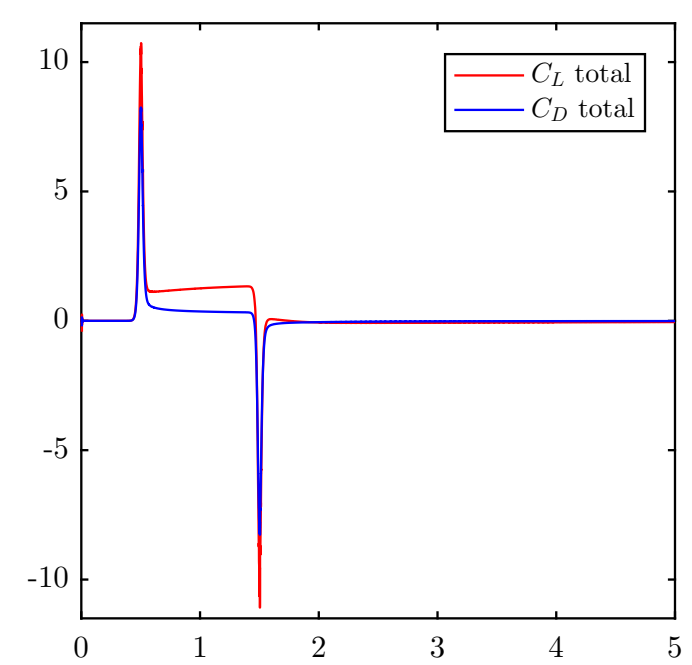

Figure 23. Drag and lift coefficients for the impulsively started and stopped airfoil. 
- The reference velocity $V_{\infty}$ was set to $1 \mathrm{~m} / \mathrm{s}$. This velocity is either the maximum free stream velocity or the maximum speed of the airfoil.

- The reference length $c$ is the chord length of the airfoil and it was set to $1 \mathrm{~m}$.

- The dynamic viscosity $\mu$ was set to $10^{-3} \mathrm{~kg} /(\mathrm{m} \cdot \mathrm{s})$ and the density was set to $1 \mathrm{~m}^{3} / \mathrm{kg}\left(v=\frac{\mu}{\rho}\right)$ to meet the Reynolds number requirement.

Using $c$ as length scale, $V_{\infty}$ as velocity scale, and $p_{\text {ref }}=$ $\rho V_{\infty}^{2}$ as pressure scale the incompressible Navier-Stokes equations with constant viscosity can be written in dimensionless form, see (White, 2011, Chapter 5) for details:

$$
\frac{D \vec{V}}{D t}=-\vec{\nabla} p+\frac{1}{R e} \nabla^{2} \vec{V}
$$

This equation shows that the only parameter present in the governing differential equations is the Reynolds number. As a consequence, the flow around the airfoil does only depend on the geometry of the airfoil (shape and angle of attack), the time variation of the inlet boundary conditions and the value of the Reynolds number (but not on the particular values of velocity, viscosity, and chord).

\section{Results and Discussion.}

\subsection{Lift and drag coefficients}

The lift and drag coefficients as a function of time are shown from Figure 18 to Figure 23. The results of the simulations when the airfoil is impulsively started and when it is stationary are shown in Figure 18 to Figure 20. The results when the airfoil motion is started and stopped are shown from Figure 21 to Figure 23. The graphs include a comparison of the viscous and pressure components of the lift and drag coefficients as well as a comparison of the overall lift and drag coefficients.

The most remarkable result is the sudden jump of the lift and drag when the acceleration occurs and the little impact that viscosity has on the value of the coefficients during this acceleration. The low influence of the viscous stresses is striking considering that, as discussed in Section 2.3, viscosity is responsible for the formation of the starting vortex and the development of the circulation that leads to the lift force in steady state.

The large drag during acceleration is not caused by the viscous stresses, instead it is an added mass effect resulting from the pressure distribution that arises when the airfoil accelerates the fluid around it (note the close relation between the acceleration peaks and the lift and drag peaks).Another interesting result is the negative peak of lift and drag when the airfoil is decelerated.

As a final remark, the variation of the lift coefficient contains higher order harmonics of small amplitude superimposed to the main variation with time.

\subsection{Verification of Kelvin's and Stoke's theo- rems}

As discussed at the end of Section 2.3, Kelvin's circulation theorem states that the circulation around an inviscid countour far away from the airfoil must be zero at all times. In addition, according to Stokes' theorem, the value of the the circulation must be given both by the line integral of velocity over the contour and the area integral of vorticity over the surface enclosed by the contour.

These two conditions where checked for the simulation of the started and stopped airfoil at $t=5 \mathrm{~s}$ using the contours shown in Figure 24. For these integration contours, the integration of Eq. 5 is given by Eq. 20 to Eq. 25, where the superscripts $V$ or $\omega$ indicate that the circulation is computed as the line integral of velocity or as the area integral of vorticity, respectively, and the subscripts net, left or right indicate the the domain of integration

$$
\begin{aligned}
& \Gamma_{\text {net }}^{V}=\oint_{\text {aefgcd }}(u, v) \cdot(d x, d y) \\
& \Gamma_{\text {left }}^{V}=\oint_{\text {abcd }}(u, v) \cdot(d x, d y) \\
& \Gamma_{\text {right }}^{V}=\oint_{\text {efgh }}(u, v) \cdot(d x, d y) \\
& \Gamma_{\text {net }}^{\omega}=\iint_{D_{1} \cup D_{2}} \omega(x, y) \cdot d x d y \\
& \Gamma_{\text {left }}^{\omega}=\iint_{D_{1}} \omega(x, y) \cdot d x d y \\
& \Gamma_{\text {right }}^{\omega}=\iint_{D_{2}} \omega(x, y) \cdot d x d y
\end{aligned}
$$

The results of the integrals are shown in Table 4 and they show excellent agreement with the theoretical results. The integrals where performed with the MATLAB builtin functions integral and quad $2 d$ using an interpolation of the OpenFOAM simulation results as the integrand at point $(x, y)$. All line integrals converged successfully to the default tolerances, but only the area integral over $D_{2}$ satisfied the tolerance criteria. The reason why the other area integrals did not converge is that the airfoil is a discontinuity of vorticity in a non-linear region that the Cartesian integration algorithms of MATLAB can not handle effectively. Despite this the values of line and area integrals are virtually identical.

Table 4. Computation of circulation.

\begin{tabular}{cccc}
\hline Type of integration & $\Gamma_{\text {net }}$ & $\Gamma_{\text {left }}$ & $\Gamma_{\text {right }}$ \\
\hline$\Gamma^{V}$ & -0.0007 & -0.5206 & 0.5199 \\
$\Gamma^{\omega}$ & 0.0009 & -0.5205 & 0.5214 \\
\hline
\end{tabular}




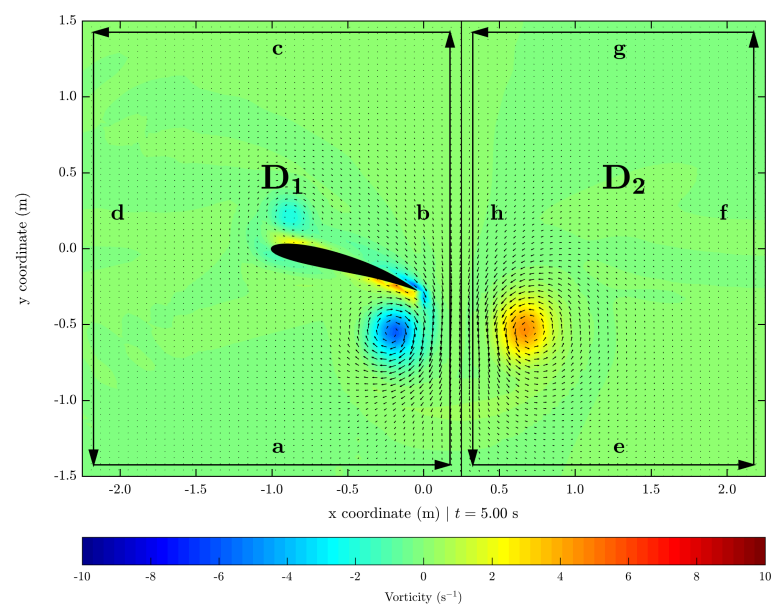

Figure 24. Integration paths for the computation of circulation.

\subsection{Flow Animations}

The results exported to MATLAB were used to prepare an animation of the three different flow configurations considered in this work. The video with the flow animations can be found in https://www. youtube. $\mathrm{com} /$ watch?v=bvV7 $-9 \mathrm{wAXc} 0$. The flow animations are qualitatively similar to the historical flow visualization from Prandtl, see (National Committee for Fluid Mechanics Films, 1972), including the formation of starting and stopping vortices as well as oscillatory vortex shedding for the case of the stationary airfoil.

\subsection{Further works}

The inclusion of the non inertial term in the Navier-Stokes equations could be considered in future works to show if its influence is indeed negligible or not. In addition, other transient effects such as the vortices shed when the angle of attack is abruptly changed could be analyzed.

\section{Conclusions}

The transient flow around a NACA4612 airoil profile was analyzed and simulated at $\operatorname{Re}=1000$ and $\alpha=16^{\circ}$. The main features of the flow were captured and the flow pattern agreed qualitatively with the flow visualizations from (National Committee for Fluid Mechanics Films, 1972).

The solution was confirmed to be grid independent and the C-mesh structured grid was found to be superior for this case as the flow was resolved with higher detail for the same number of cells.

Once the lift and drag coefficients were computed it was found that both peaked during the accelerations and decelerations due to added mass effects and that the effect of the viscous stresses on lift was modest even if viscosity is responsible for the development of lift. In addition, the solution was verified using the Kelvin's circulation theorem. Both the line integral of the velocity and the surface integral of vorticity matched with reasonable accuracy and were almost equal to zero for an inviscid contour and nonzero for viscid contours.

\section{References}

G. K. Batchelor. An Introduction to Fluid Dynamics. Cambridge Mathematical Library, 1st edition, 2000.

D. Darmofal. Aerodynamics, fall 2005, 2001. URL https : //ocw.mit.edu.

R. W. Fox, P. J. Pritchard, and A. T. McDonald. Fox and McDonald's Introduction to Fluid Mechanics. John Wiley \& Sons, 9th edition, 2011.

E. N. Jacobs, K. E. Ward, and R. M. Pinkerton. The characteristics of 78 related airfoil sections from tests in the variabledensity wind tunnel. National Advisory Committee for Aeronautics Report No. 460, 1933.

A. Jeffrey. Advanced Engineering Mathematics. Academic Press, 1st edition, 2001.

P. K. Kundu and I. M. Cohen. Fluid Mechanics. Academic Press, 2th edition, 2002.

National Committee for Fluid Mechanics Films. Illustrated experiments in fluid mechanics: the NCFMF book of film notes, Vorticity, 1972. URL http://web.mit.edu/ $\mathrm{hml} / \mathrm{ncfmf} . \mathrm{html}$.

M. Vincent and H. M. Blackburn. Simulation of starting/stopping vortices for a lifting aerofoil. Proceedings of the 19th Australasian Fluid Mechanics Conference, 2014.

F. M. White. Viscous Fluid Flow. McGraw-Hill, 3th edition, 2006.

F. M. White. Fluid Mechanics. McGraw-Hill, 7th edition, 2011.

C. Willert and J. Kompenhans. PIV Analysis of Ludwig Prandtl's Historic Flow Visualization Films. arXiv preprint arXiv:1010.3149, 2010. 